\title{
PROJECTIVE INVARIANTS OF INTERSECTION OF CERTAIN PAIRS OF SURFACES
}

\section{CHUAN-CHIH HSIUNG}

1. Introduction. In a recent paper $[2]^{1}$ the author has shown the existence, together with metric and projective characterizations, ${ }^{2}$ of a unique projective invariant determined by the neighborhood of the second order of two surfaces $S_{1}, S_{2}$ at an ordinary point $O$ in ordinary space when the tangent planes $\tau_{1}, \tau_{2}$ of the surfaces $S_{1}, S_{2}$ at the point $O$ are distinct and the line $t$ of intersection of the two tangent planes $\tau_{1}, \tau_{2}$ does not coincide with any one of the asymptotic tangents of the surfaces $S_{1}, S_{2}$ at the point $O$. On the other hand, with regard to the coincidences of the line $t$ and the asymptotic tangents of the surfaces $S_{1}, S_{2}$ at the point $O$ two essentially different cases can arise. The object of this note is to derive some projective invariants in these cases. Noticing that no projective invariant can be determined by the neighborhood of the second order of the surfaces $S_{1}, S_{2}$ at the point $O$, we obtain all projective invariants determined by the neighborhood of the second order of one surface and that of the third order of the other at the point $O$.

\section{Two SURFACES WITH DISTINCT TANGENT PLANES AND DISTINCT ASYMPTOTIC TANGENTS AT AN ORDINARY POINT}

2. Derivation of invariants. Let us first consider two surfaces $S_{1}, S_{2}$ in ordinary space intersecting at an ordinary point $O$ with distinct tangent planes $\tau_{1}, \tau_{2}$, whose line of intersection $t$ is supposed to be an asymptotic tangent of the surface $S_{1}$ at the point $O$. Let $t_{1}$ be the other asymptotic tangent of the surface $S_{1}$ at the point $O$, and $t_{2}$ the harmonic conjugate line of $t$ with respect to the asymptotic tangents of the surface $S_{2}$ at the point $O$. If we choose the point $O$ to be the origin, the lines $t, t_{2}, t_{1}$ to be respectively the axes $x, y, z$ of a general nonhomogeneous projective coordinate system, then the power series expansions of the surfaces $S_{1}, S_{2}$ in the neighborhood of the point $O$ may be written in the form

$$
\begin{array}{ll}
S_{1}: & y=l x z+p x^{3}+r x^{2} z+s x z^{2}+q z^{3}+\cdots, \\
S_{2}: & z=m x^{2}+n y^{2}+\cdots .
\end{array}
$$

Received by the editors January 26, 1944.

${ }_{1}^{1}$ Numbers in square brackets refer to the references at the end of the paper.

${ }^{2}$ An extension of these results to two hypersurfaces has been made by Professor Su. See his paper [6]. 
In order to find projective invariants of the surfaces $S_{1}, S_{2}$ at the point $O$, we have to consider the most general projective transformation of coordinates which shall leave the lines $t, t_{1}, t_{2}$ unchanged:

$$
\begin{aligned}
& x=a_{22} x^{*} /\left(1+a_{12} x^{*}+a_{13} y^{*}+a_{14} z^{*}\right), \\
& y=a_{33} y^{*} /\left(1+a_{12} x^{*}+a_{13} y^{*}+a_{14} z^{*},\right. \\
& z=a_{44} z^{*} /\left(1+a_{12} x^{*}+a_{18} y^{*}+a_{14} z^{*}\right),
\end{aligned}
$$

where $a_{i k}$ are arbitrary constants. The effect of this transformation on equations (1), (2) is to produce two other equations of the same form whose coefficients, indicated by stars, are given by the formulas

$$
\begin{gathered}
a_{33} z^{*}=a_{22} a_{44} l, \quad a_{33} p^{*}=a_{22}^{8} p, \quad a_{33} *^{*}=a_{44}^{8} q, \\
2 a_{12} a_{33} l^{*}+a_{33} r^{*}=a_{12} a_{22} a_{44} l+a_{22}^{2} a_{44} r, \\
2 a_{14} a_{33} l^{*}+a_{33} s^{*}=a_{14} a_{22} a_{44} l+a_{22} a_{44} s ; \\
a_{44} m^{*}=a_{22}^{2} m, \quad a_{44} n^{*}=a_{38}^{2} n .
\end{gathered}
$$

Further elimination of $a_{i k}$ from equations (4) gives immediately that the quantities

$$
I=l m / p, \quad J=p^{2} q^{4} m^{8} / l^{12} n^{8}
$$

are projective invariants associated with the surfaces $S_{1}, S_{2}$, at the point $O$.

3. Projective characterizations of the invariants $I, J$. It is well known that the tangent plane $\tau_{1}$ intersects the surface $S_{1}$ in a curve with a node at the point $O$, the nodal tangents being the asymptotic tangents $t, t_{1}$. The expansion of the branch $C_{1}$ of this curve which is tangent to the tangent $t, z=y=0$, is easily found to be

$$
z=-(p / l) x^{2}+\cdots \text {. }
$$

On the other hand, the curve $C_{2}$ in which the tangent plane $\tau_{1}$ intersects the surface $S_{2}$ is given by the equations

$$
z=m x^{2}+\cdots, \quad y=0 .
$$

It is thus easy to reach the conclusion that the projective invariant $I$ associated with the point $O$ of the surfaces $S_{1}, S_{2}$ is, except for sign, equal to the projective invariant of contact ${ }^{3}$ of the two plane curves $C_{1}, C_{2}$ at the point $O$.

To characterize projectively the other invariant $J$ it is useful to

${ }^{3}$ The projective invariant of contact of two plane curves having ordinary contact at a point was first found by H. J. S. Smith [5] and R. Mehmke [3]; and its simple projective characterization was later given by C. Segre [4]. 
consider the asymptotic curves $\Gamma, \Gamma_{1}$ of the surface $S_{1}$ at the point $O$. The expansions of these asymptotic curves at the point $O$ are found, after a simple calculation, to be

$$
\begin{array}{lll}
\Gamma: & y=-2 p x^{3}+\cdots, & z=-(3 p / l) x^{2}+\cdots ; \\
\Gamma_{1}: & y=-2 q z^{3}+\cdots, & x=-(3 q / l) z^{2}+\cdots .
\end{array}
$$

Let $K$ be any five-point quadric cone of the asymptotic curve $\Gamma$ at the point $O$ with $O$ for vertex and with an asymptotic tangent of the surface $S_{2}$ at the point $O$ for a generator, then making use of equations (8) we can easily obtain its equation, namely,

$$
z^{2}+\left(9 p / 2 l^{2}\right) x y \pm\left(9 p / 2 l^{2}\right)(-n / m)^{1 / 2} y^{2}+k x z=0,
$$

where $k$ is arbitrary. Similarly, we have a five-point quadric cone $K_{1}$ of the asymptotic curve $\Gamma_{1}$ at the point $O$ such that it has $O$ for vertex and the line $t_{2}$ for a generator and is tangent to the plane $\tau_{2}$, $z=0$, along $t_{2}$. The equation of this cone $K_{1}$ is

$$
x^{2}+\left(9 q / 2 l^{2}\right) y z=0 \text {. }
$$

From equations (10), (11) it follows that the cone projecting the curve of intersection of the cones $K, K_{1}$ from the line $t_{2}$ consists of the four planes:

$$
9 q^{2} z^{4}-18 k l^{2} q x^{2} z^{2}-9 p q x^{3} z \pm 2 l^{2} p(-n / m)^{1 / 2} x^{4}=0,
$$

which are intersected by the plane $\tau_{1}$ in four lines $\lambda_{i}(i=1, \cdots, 4)$ with equations

$$
z+\alpha_{i} x=0, \quad y=0 \quad(i=1, \cdots, 4),
$$

where the coefficients $\alpha_{i}$ satisfy the relation

$$
\alpha_{1} \alpha_{2} \alpha_{3} \alpha_{4}= \pm\left(2 l^{2} p / 9 q^{2}\right)(-n / m)^{1 / 2} .
$$

On the other hand, associated with the point $O$ of the surface $S_{1}$ there are three tangents of Darboux, whose equations may be found by making use of equations (8), (9) and Bompiani's result [1] that they are the three principal lines of the asymptotic curves $\Gamma, \Gamma_{1}$ at the point $O$. The result is

$$
p x^{3}+q z^{3}=0, \quad y=0 .
$$

Let $d$ be any one of the tangents (15) of Darboux, and let $D_{i}$ denote the cross ratio of the four lines $t, t_{1}, d, \lambda_{i}(i=1, \cdots, 4)$; then

$$
D_{i}=\left(t t_{1}, d \lambda_{i}\right)=\left(1 / \alpha_{i}\right)(p / q)^{1 / 3} \quad(i=1, \cdots, 4) .
$$


A reference to equations (5), (14), (16) suffices to substantiate that the invariant $J$ can be expressed in terms of the four cross ratios $D_{1}, D_{2}$, $D_{3}, D_{4}$ as follows:

$$
J=-(2 / 9)^{6}\left(D_{1} D_{2} D_{3} D_{4}\right)^{6}
$$

II. Two SURFACES WITH DISTINCT TANGENT PLANES AND A COMMON ASYMPTOTIC TANGENT AT AN ORDINARY POINT

4. Derivation of an invariant. Finally, suppose that $S_{1}, S_{2}$ be two surfaces in ordinary space intersecting at an ordinary point $O$ with distinct tangent planes $\tau_{1}, \tau_{2}$, whose line of intersection $t$ stands for one asymptotic tangent of both surfaces $S_{1}, S_{2}$ at the point $O$; and that $t_{1}, t_{2}$ be respectively the other asymptotic tangents of the surfaces $S_{1}, S_{2}$ at the point $O$. If we choose the point $O$ to be the origin, the lines $t, t_{2}, t_{1}$ to be respectively the axes $x, y, z$ of a general nonhomogeneous projective coordinate system, then the power series expansions of the surfaces $S_{1}, S_{2}$ in the neighborhood of the point $O$ may be written in the form (1) and

$$
S_{2}: \quad z=m x y+\cdots .
$$
tity

In a way similar to the foregoing we can easily show that the quan-

$$
I=p^{4} q^{2} / l^{9} m^{3}
$$

is a projective invariant associated with the surfaces $S_{1}, S_{2}$ at the point $O$.

5. A projective characterization of the invariant $I$. Let $\Gamma, \Gamma_{1}$ be the asymptotic curves of the surface $S_{1}$ at the point $O$ whose tangents are $t, t_{1}$ respectively. Among the four-point quadrics of the asymptotic curve $\Gamma$ at the point $O$ we can determine a two-parameter family such that every one of the family has $t_{2}$ for a generator and has at the point $O$ contact of the second order with the surface $S_{2}$. By means of equations (8), (18) it is easy to obtain the equation of a general quadric of this family, namely,

$$
z+(3 p / l) x^{2}-m x y+E y z+F z^{2}=0,
$$

where $E, F$ are arbitrary. The quadric (20) is cut by the plane $\tau_{2}$ in the line $t_{2}$ and a line with equations

$$
3 p x-\operatorname{lm} y=0, \quad z=0 .
$$

If any five-point quadric cone, with the point $O$ for vertex, of the 
asymptotic curve $\Gamma$ at $O$ passes through the line (21), then it must have the equation:

$$
z^{2}+\left(9 p / 2 l^{2}\right) x y-(3 m / 2 l) y^{2}+k y z=0,
$$

where $k$ is arbitrary. The cone projecting the curve of intersection of the cones (11), (22) from the line $t_{2}$ consists of the four planes:

$$
27 q^{2} z^{4}-6 k l^{2} q x^{2} z^{2}-27 p q x^{3} z-2 l^{3} m x^{4}=0,
$$

which are intersected by the plane $\tau_{1}$ in four lines $\mu_{i}(i=1, \cdots, 4)$ :

$$
z+\beta_{i} x=0, \quad y=0 \quad(i=1, \cdots, 4),
$$

where the coefficients $\beta_{i}$ satisfy the relation

$$
\beta_{1} \beta_{2} \beta_{3} \beta_{4}=-2 l^{3} m / 27 q^{2} .
$$

If $d$ be any one of the three tangents of Darboux associated with the point $O$ of the surface $S_{1}$ and if $D_{i}$ denotes the cross ratio of the four lines $t, t_{1}, d, \mu_{i}(i=1, \cdots, 4)$, then from equations (15), (24) it follows that

$$
D_{i}=\left(t t_{1}, d \mu_{i}\right)=\left(1 / \beta_{i}\right)(p / q)^{1 / 3} \quad(i=1, \cdots, 4) .
$$

A reference to equations (19), (25), (26) suffices to show that the invariant $I$ can be expressed in terms of the four cross ratios $D_{1}, D_{2}, D_{3}$, $D_{4}$ as follows:

$$
I=-(2 / 27)^{3}\left(D_{1} D_{2} D_{3} D_{4}\right)^{3}
$$

\section{REFERENCES}

1. E. Bompiani, Invarianti d'intersezione di due curve sghembe, Rendiconti dei Lincei (6) vol. 14 (1931) pp. 456-461.

2. C. C. Hsiung, An invariant of intersection of two surfaces, to be published in the American Journal of Mathematics.

3. R. Mehmke, Einige Sätze ìber die räumliche Collineation und Affinität, welche sich auf die Krilmmung von Curven und Flächen beziehen, Schlömilchs Zeitschrift für Mathematik und Physik vol. 36 (1891) pp. 56-60; Über zwei die Krilmmung von Curven und das Gauss'sche Krïmmungsmass von Flächen betreffende charakteristische Eigenschaften der linearen Punkttransformationen, ibid. vol. 36 (1891) pp. 206-213.

4. C. Segre, Su alcuni punti singolari delle curve algebriche, e sulla linea parabolica di una superficie, Rendiconti dei Lincei (5) vol. 6 (1897) pp. 168-175.

5. H. J. S. Smith, On the focal properties of homographic figures, Proc. London Math. Soc. vol. 2 (1869) pp. 196-248.

6. B. Su, A new invariant of intersection of two hypersurfaces, to be published in the Revista, Universidad Nacional de Tucumán (A).

National University of Chekiang 